$$
\text { إعادة استخدام مياه الصرف الصحي المعالجة بمفاعل الأغشية الحيوية في الري }
$$

\title{
Reusing of Treated Wastewater by Membrane Bioreactorin Irrigation
}

$$
\text { طهري شنشل جعفر }
$$

Azzam H. Al-Hadithy

A.A. Hasson

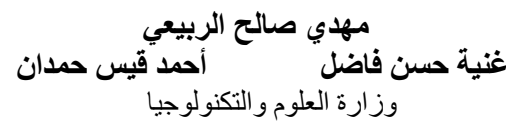 \\ Mahdi S. Al-Rubai
}

G.H. FadeL A.Q. Hamdn

Ministry Of Science and Technology

\author{
عزام حمودي الحليثي \\ إلهام عبد المثلك حسون \\ Mahdi Sh. Jaafar \\ T.R. Edan
}

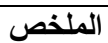

تعتبر تقنية المعالجة بطريقة مفاعلات الاغشية الحيوية MBR من احلث الوسائل والتقتيات الحديثة المستخدمة حاليا في معالجة

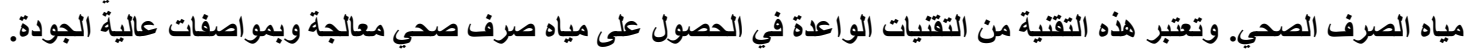

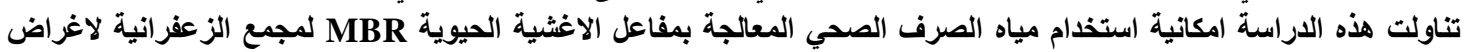

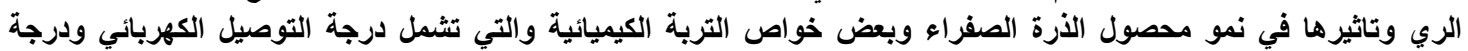

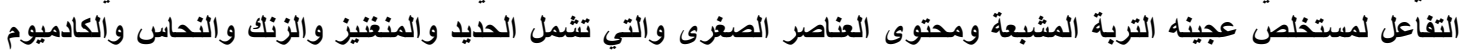

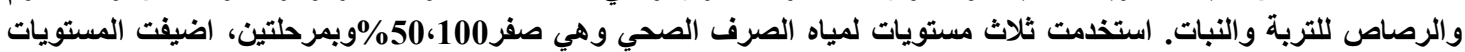

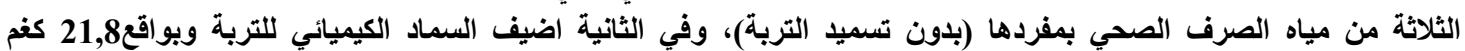


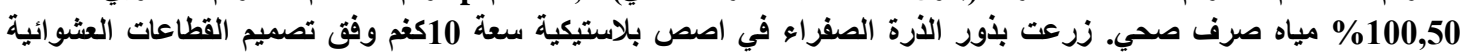

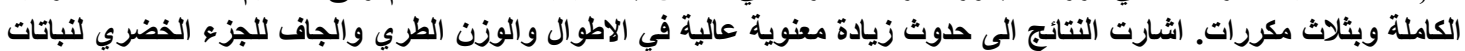

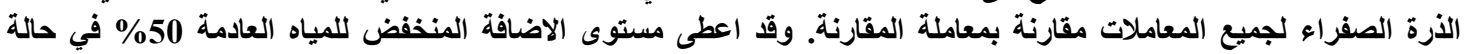

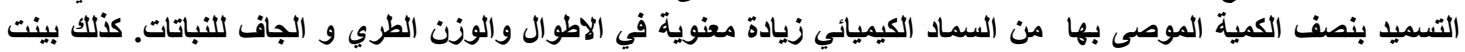

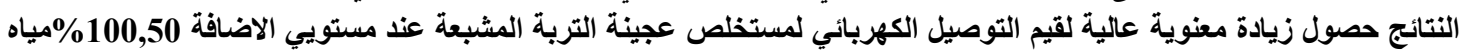

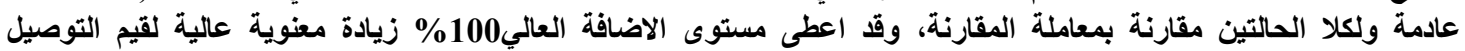

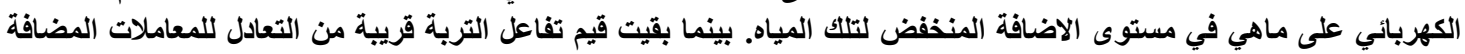

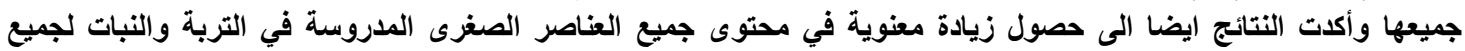

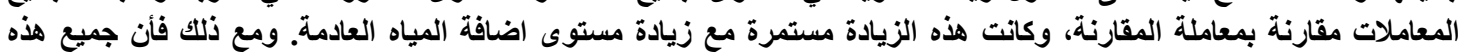

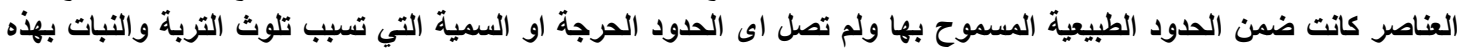

العناصر.

الكلمات المفتاحية: مياه الصرف الصحي، الأغثية الحيوية، بذور الذرة الصفراء

Abstract:

Membrane Bio Reactor(MBR) methods is considered as one of the most advanced used technology in waste water treatment. The aim of this technology is to obtain high quality treated waste water. This study conducted to investigate the ability of using of sewage water from zaafrania city for irrigation and their effect on corn growth and some of soil chemical properties which include electrical conductivity and soil $\mathrm{pH}$ in extract soil paste and the micro nutrient content in soil and plant which include $\mathrm{Fe}, \mathrm{Mn}, \mathrm{Zn}, \mathrm{Cu}, \mathrm{Cd}, \mathrm{Pb}$. Three levels of wastewater were used $0,50,100 \%$ in two stages, the three levels of wastewater ( without soil fertilization ) were used in the first stage, where $21.8 \mathrm{Kg} \mathrm{P} / \mathrm{D}+80 \mathrm{~N} \mathrm{Kg} / \mathrm{D}$ was added to the soil as fertilizer in the control $0 \%$ treatment and $10.9 \mathrm{Kg} \mathrm{P} / \mathrm{D}+40 \mathrm{Kg} \mathrm{N} / \mathrm{D}$ were added to 50 and $100 \%$ levels in the second stage. Corn seeds were planted in $10 \mathrm{~kg}$ plastic pots in Completely Randomized Block Design in three replicates. The results refer to a high significant increase in plant height, fresh and dry weight for all treatments in comparison with control treatment. The low add level of wastewater $50 \%$ + half of fertilizer recommendation gives a significant increase of plant height and fresh and dray weight. The results showed a high increased of electrical conductivity for 50, $100 \%$ wastewater added levels for both stages compared with control treatment. The high add level $100 \%$ gives high significant increase of electrical conductivity compared with the low level of the wastewater. Whereas the values of soil $\mathrm{PH}$ were close to the neutral for all treatment. The result showed a significant increase in micro nutrients content (which include $\mathrm{Fe}, \mathrm{Mn}, \mathrm{Zn}, \mathrm{Cu}, \mathrm{Cd}$, $\mathrm{Pb}$ ) in soil and plant for all treatment compared with control treatment, this increased was 
continues with the increase of additional level of wastewater. However all the micro nutrient was within the allowed natural limits and not reached the toxic limits in soil and plant.

Key words: Wastewater, Membrane Bioreactor, Corn seeds

المقدمة

برزت قضية شحة المياه وتردي نو عيتها في العديد من الدول نتيجة النمو السكاني و التغير ات المناخية و النشاط الأنساني مما سبب فجوة

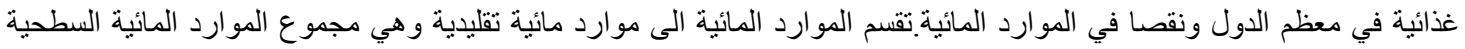

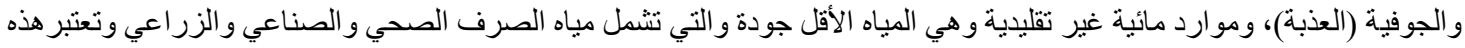

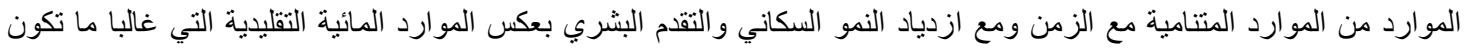

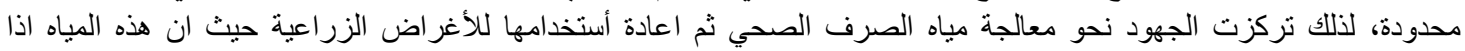

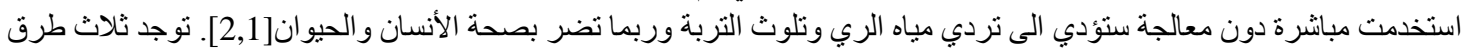

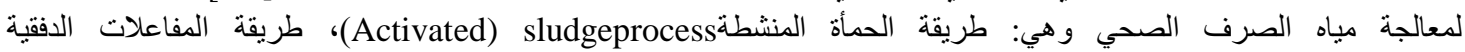

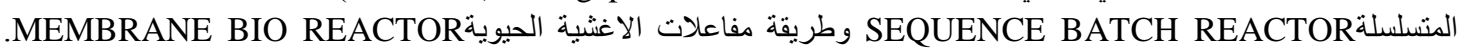

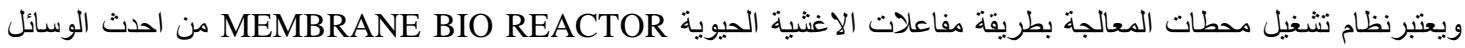

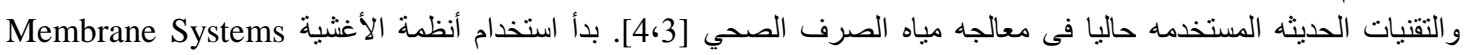

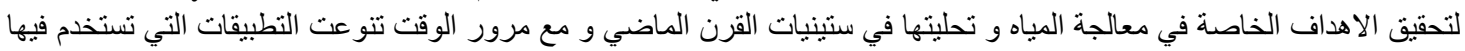

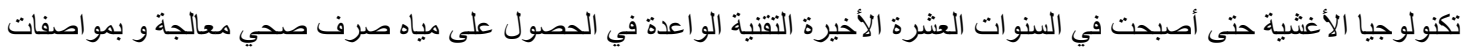

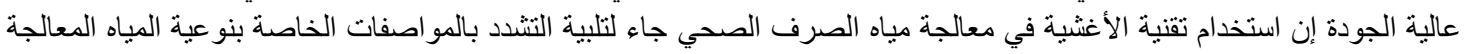

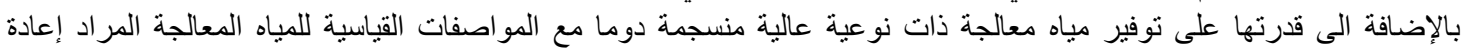

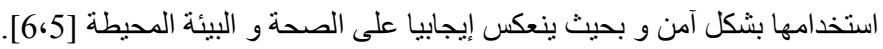

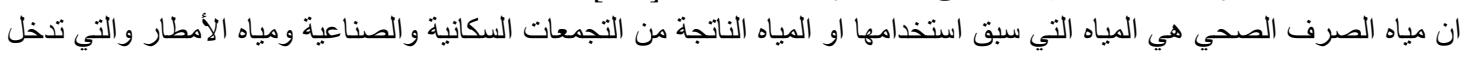

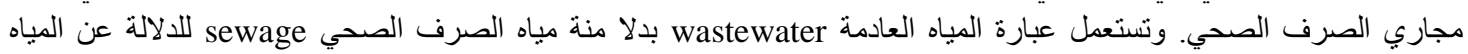

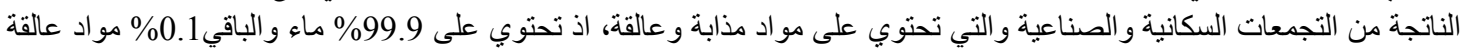

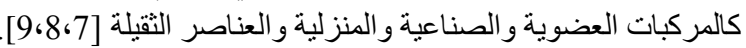

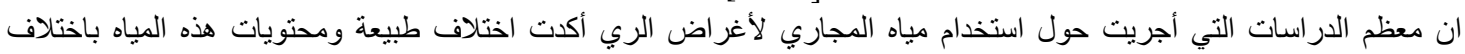

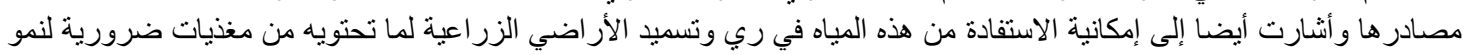

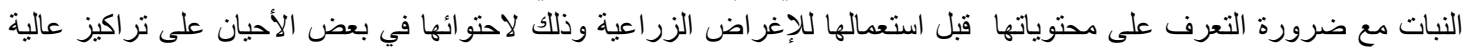

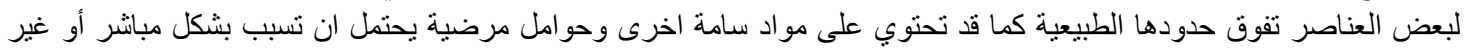

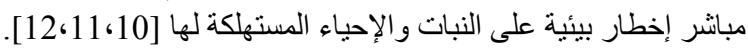

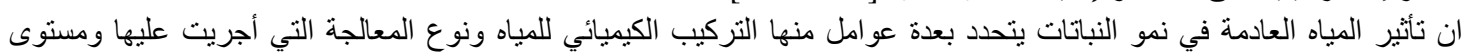

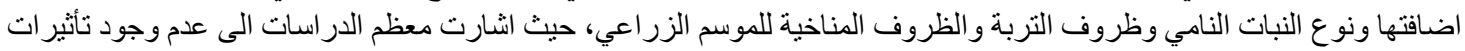

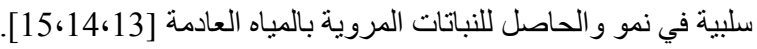

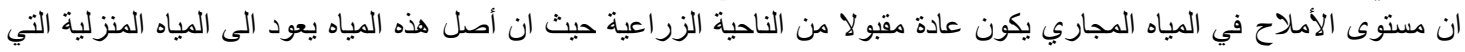

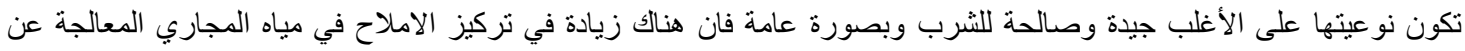

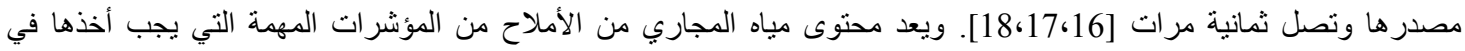

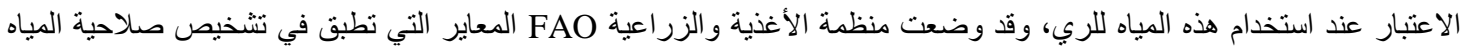

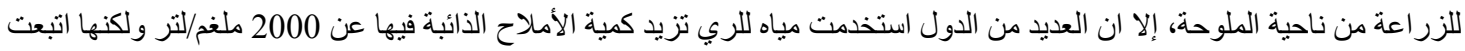

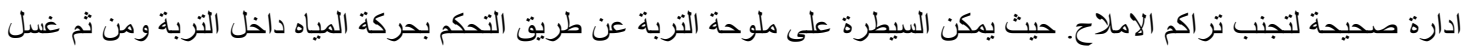

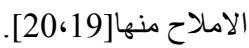
تشكل محتويات مباه المجاري من العناصر الثقيلة مصدر قلق نظر التأثثير ها على خواص كل من التربة والنبات و المياه الجوفية والبيئة

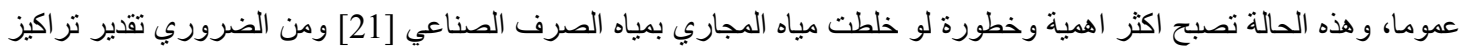

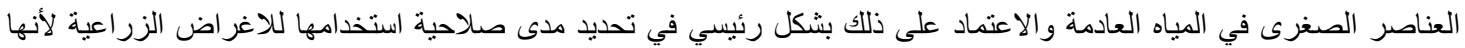

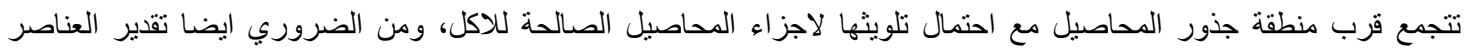

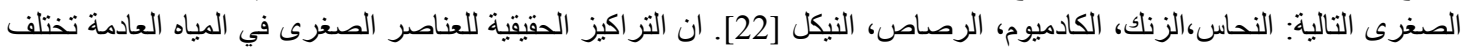

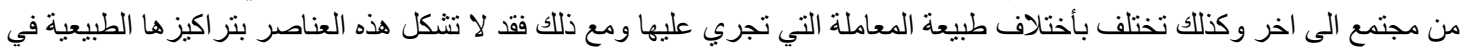

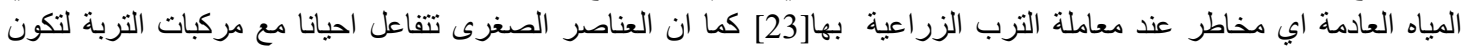

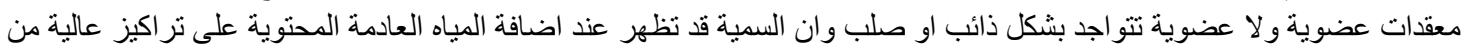

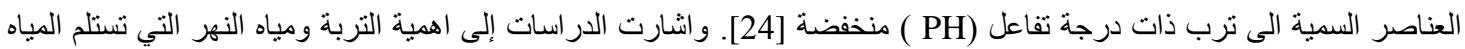

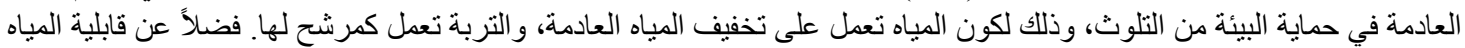




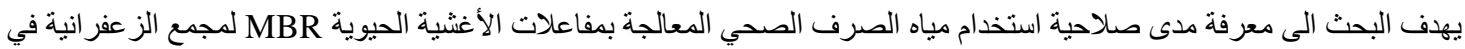
ري محصول الذرة الصفر اء وتأثير ها في بعض خو اص التربة الكيمياوية ومحتوى العناصر الثقيلة في التربة والنبات.

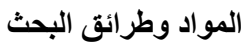

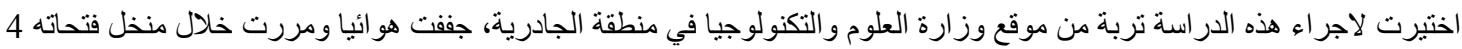

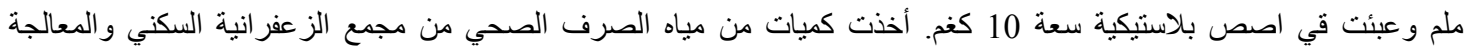

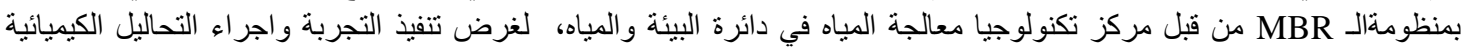

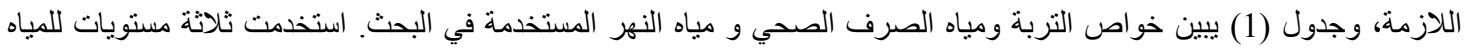

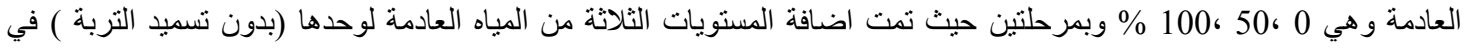

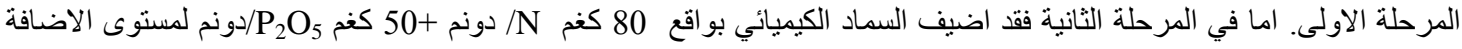

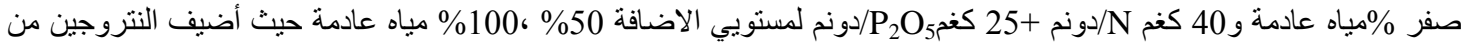

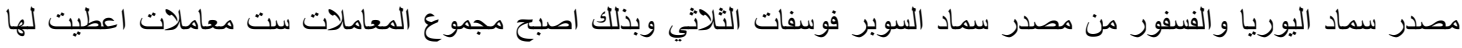

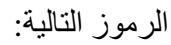

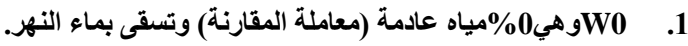

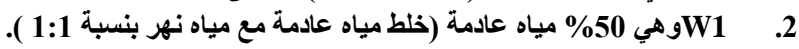
ك

( W0+F

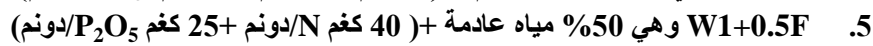

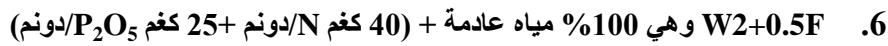

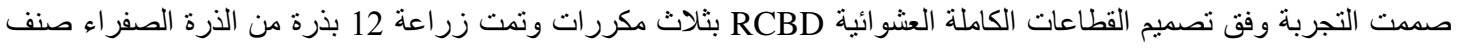

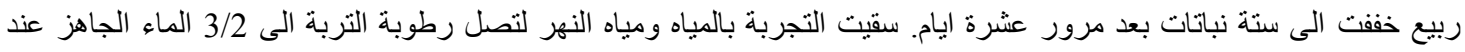

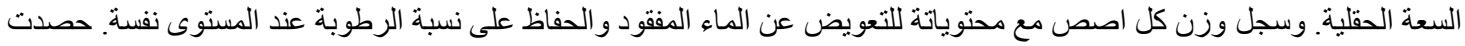

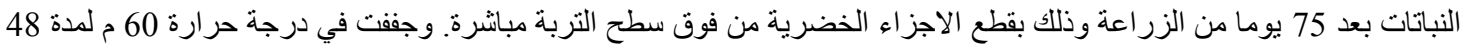

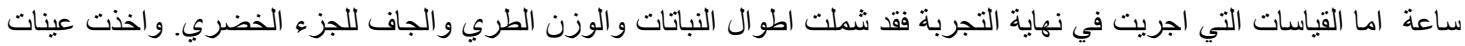

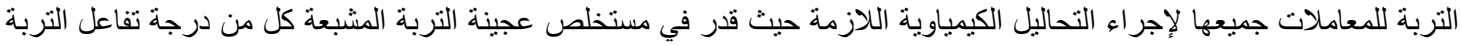

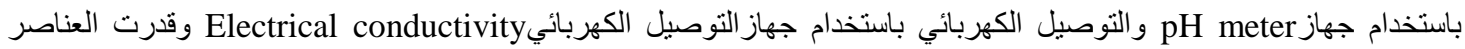
DTPADiethylenTriaminPenta Acetic الصغرى وذلك بتحضير مستخلص تربة برج 10ml منها في 20ml من محلول

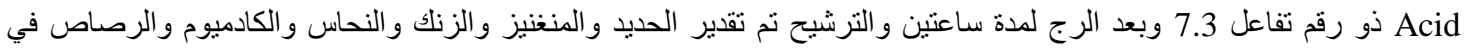
المحلول باستخدام جهاز الامتصاص الذرئ فاعل

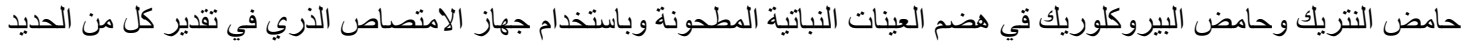

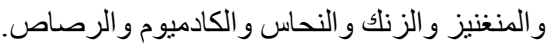

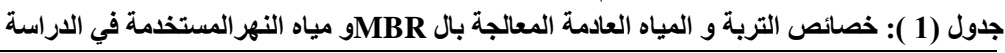

\begin{tabular}{|c|c|c|c|c|}
\hline \multirow[t]{5}{*}{ مياه النهز } & المياه العادمة & تربة & \multicolumn{2}{|r|}{ الخاصية } \\
\hline & 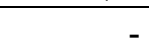 & مزيجة طينية & & النسجة \\
\hline & - & 355 & (-م-1-1) & الطين ( غم .ك \\
\hline & - & 390 & فم-1- (1-) & الغرين ( غم . \\
\hline & - & 255 & (1- 1 & الرمل ( غم .ك \\
\hline 0.93 & 1.768 & 4.0 & يسيسيمنز/م ) & التوصيل الكهربائي ) \\
\hline 8.03 & 776 & 7.45 & بية & الدالة الحامد \\
\hline & & 282 & فم-1) & الكلس ( غم .5 \\
\hline 0.56 & 24.5 & 270 & لفم .كغم-1) & البوتاسيوم الجاهز) ( الجاهن \\
\hline 0.29 & 5.3 & 6.5 & لغم .كغم-1) & الفوسفور الجاهز ( ) \\
\hline 0.45 & 3.5 & 13.5 & لـفم .كغم-1) & النترات - نتروجين() \\
\hline 0.36 & 3.5 & 9.9 & ملغم .كغק-1 ) & الأمونيوم - نتروجين) \\
\hline 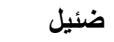 & 1.3 & 1.3 & $\mathrm{Cu}$ & \\
\hline 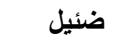 & 2.0 & 2.0 & $\mathbf{Z n}$ & \\
\hline 0.02 & 5.0 & 5.0 & Mn & العناصر الصغرى المستخلصة بـ \\
\hline 0.45 & 6.6 & 6.0 & $\mathbf{F e}$ & Dلغف .كفק-1DTPA \\
\hline ضئيل & 0.10 & 0.10 & pb & \\
\hline ضئيل & 0.22 & 0.22 & Cd & \\
\hline
\end{tabular}

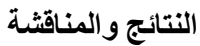

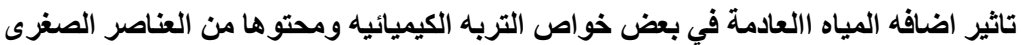

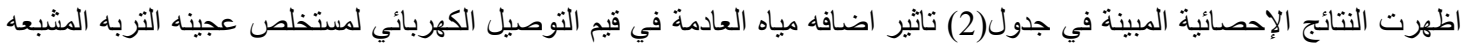
حيث نلاحظ ان هناك زيادة في درجة التوصيل الكهربائي لمستخلص عجينة التربة المشبعة مع زيادة مستوى أضافة المياه العادمة ولكلتا 


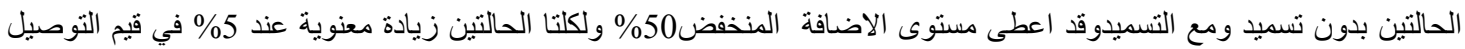

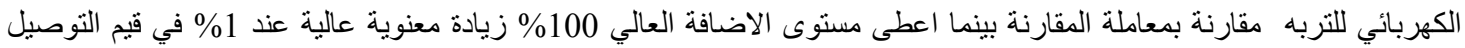

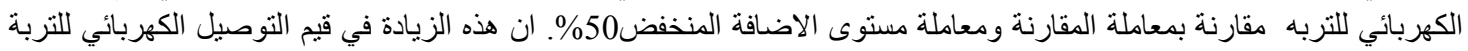

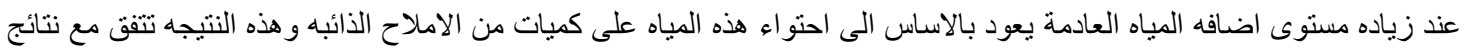

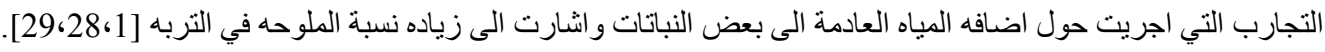

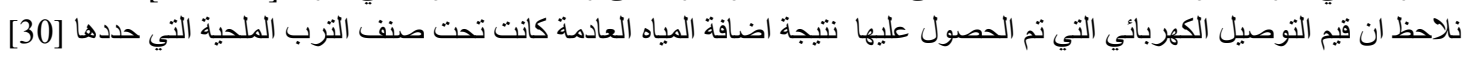

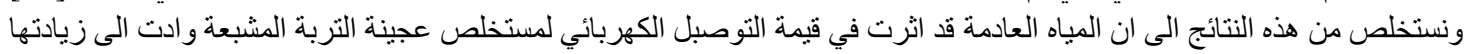

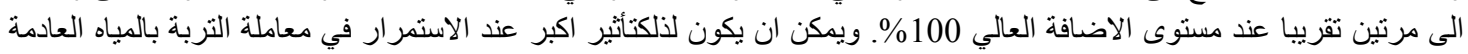

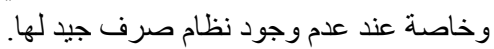

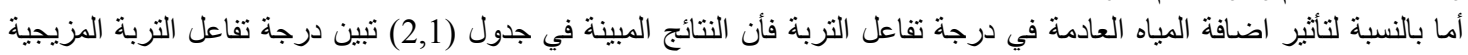

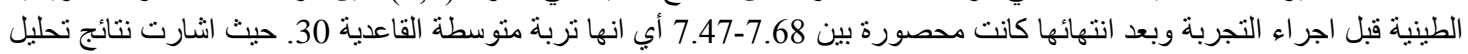

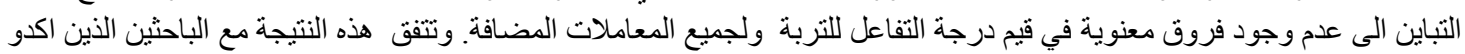

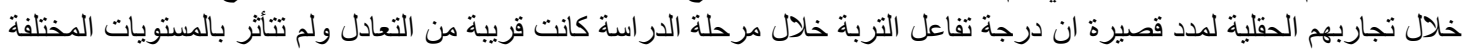

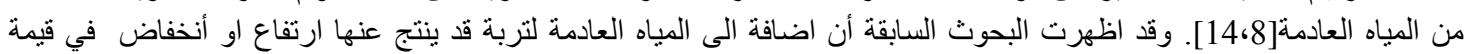

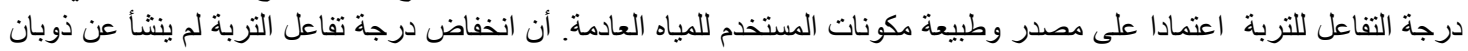

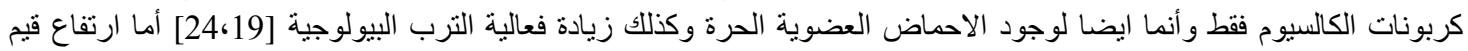

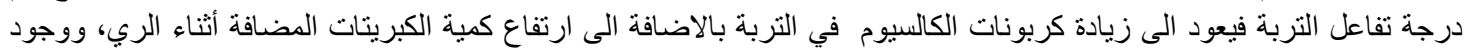

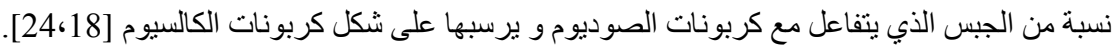

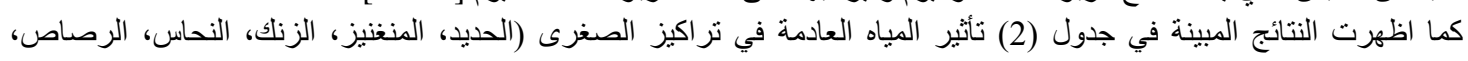

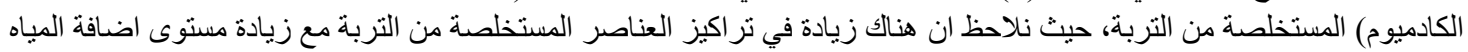

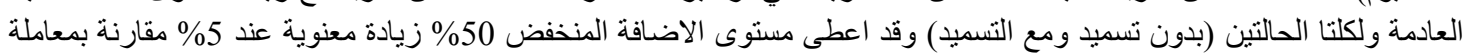

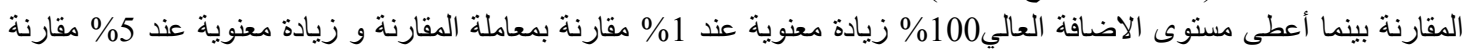

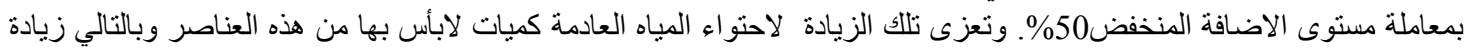

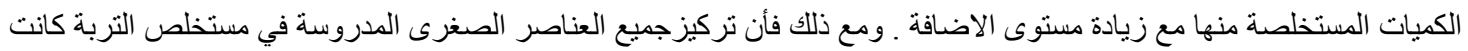

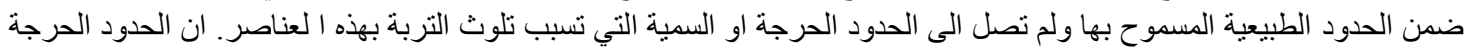

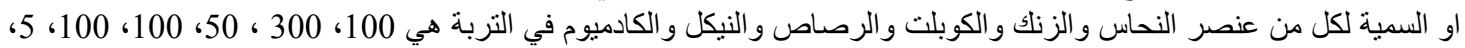

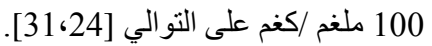

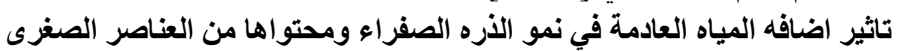

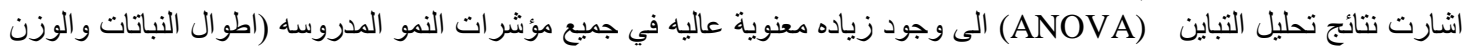

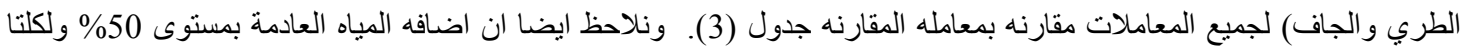

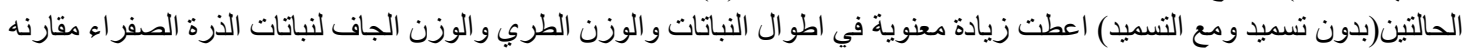

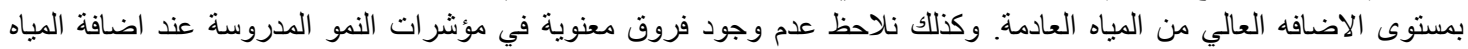

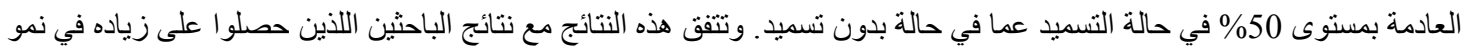

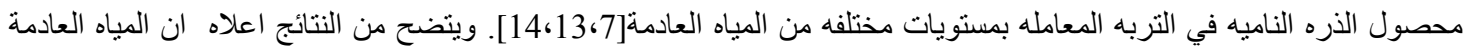

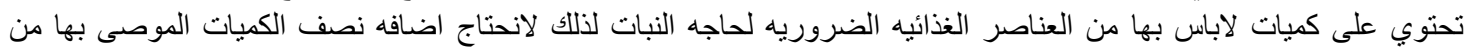

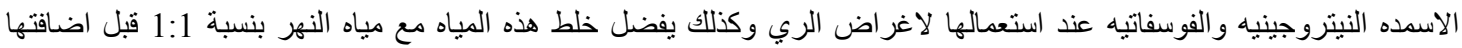

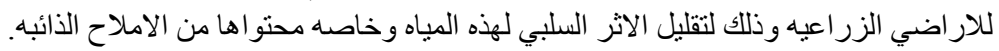

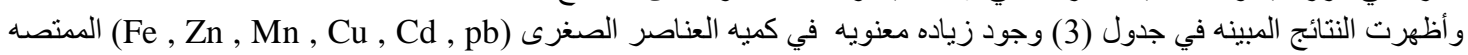

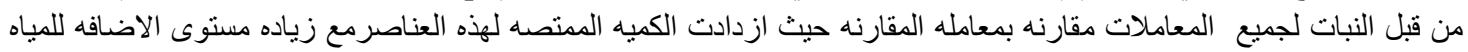

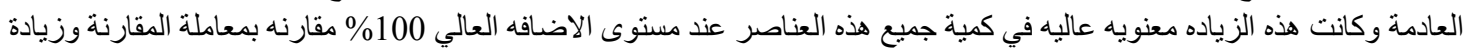

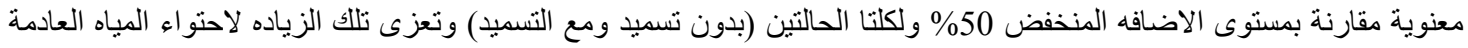

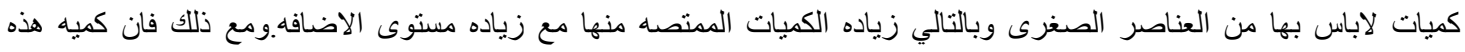

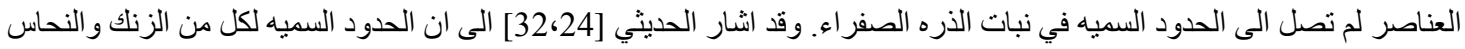

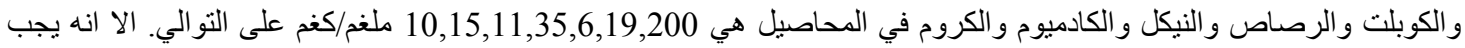

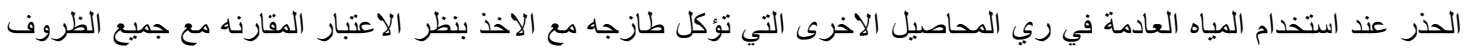

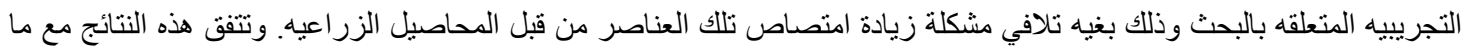

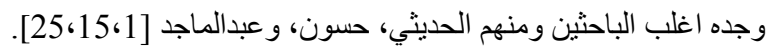

الاستنتاجات

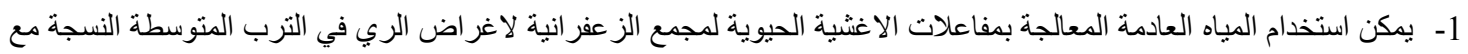

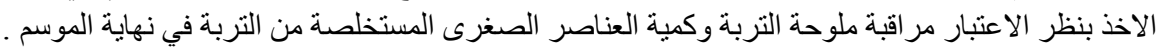


2- يفضل خلط هذه المياه مع النهر بنسبة 1:1 التقليل كمية الاملاح الذائبة فيها.

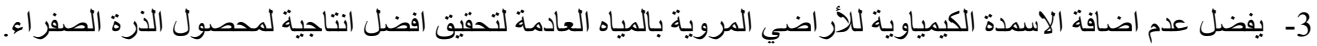

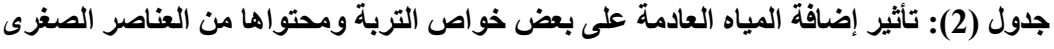

\begin{tabular}{|c|c|c|c|c|c|c|c|c|}
\hline \multicolumn{6}{|c|}{ العناصر الثقيلة المستخلصة من التربة( ملغم .كفم-1) } & \multirow{2}{*}{ درجة التفاعل } & \multirow{2}{*}{ (التوصيسل الكهربائي } & \multirow{2}{*}{ 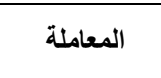 } \\
\hline $\mathbf{P b}$ & Cd & $\mathbf{C u}$ & $\mathbf{Z n}$ & Mn & $\mathbf{F e}$ & & & \\
\hline 2.0 & 0.15 & 4.3 & 10.2 & 20.7 & 11.6 & 7.70 & 3.8 & \%0 (W0) \\
\hline 2.2 & 0.18 & 5.6 & 11.8 & 23.8 & 14.9 & 7.67 & 5.2 & $\% 50$ (W1) \\
\hline 2.4 & 0.22 & 6.9 & 13.3 & 27.9 & 17.5 & 7.61 & 7.1 & \%100 (W2) \\
\hline 2.1 & 0.16 & 4.2 & 11.6 & 22.6 & 12.2 & 7.73 & 3.9 & W0+F́ \\
\hline 2.3 & 0.14 & 6.2 & 12.0 & 25.9 & 15.4 & 7.68 & 5.3 & $\mathrm{~W} 1+0.5 \mathrm{~F}$ \\
\hline 2.6 & 0.23 & 7.15 & 13.9 & 29.6 & 18.3 & 7.64 & 7.3 & $\mathrm{~W} 2+0.5 \mathrm{~F}$ \\
\hline 0.181 & $\mathbf{0 . 0 3 0}$ & 1.180 & 1.270 & 2.962 & 2.512 & 0.110 & 0.910 & $\mathrm{LSD} \leq \mathbf{0 . 0 5}$ \\
\hline 0.315 & 0.062 & 2.500 & 3.061 & 6.890 & 4.091 & 0.201 & 1.821 & $\mathrm{LSD} \leq \mathbf{0 . 0 1}$ \\
\hline
\end{tabular}

جدول (3): تأثير إضافة المياه العادمة في نمو الذرة الصفراء ومحتواها من العناصر الصغرى .

\begin{tabular}{|c|c|c|c|c|c|c|c|c|c|}
\hline \multicolumn{6}{|c|}{ محتوى العناصر الصغرى ( ملغم .كفم-1) } & \multirow{2}{*}{ الجاف(غم الوزن } & \multirow{2}{*}{ الطرزي } & \multirow{2}{*}{$\begin{array}{c}\text { طولالنبات } \\
\text { (سم) }\end{array}$} & \multirow{2}{*}{ المعاملة } \\
\hline $\mathbf{P b}$ & Cd & $\mathbf{C u}$ & $\mathbf{Z n}$ & Mn & $\mathbf{F e}$ & & & & \\
\hline 2.2 & 0.20 & 6.4 & 21 & 30 & 52 & 28 & 90 & 76 & $\% 0(\mathrm{W0})$ \\
\hline 2.6 & 0.26 & 8.0 & 29 & 41 & 59 & 44 & 198 & 118 & $\% 50$ (W1) \\
\hline 2.9 & 0.35 & 9.6 & 37 & 54 & 70 & 41 & 155 & 104 & $\% 100$ (W2) \\
\hline 2.4 & 0.25 & 7.0 & 25 & 36 & 61 & 40 & 185 & 114 & $\mathbf{W 0 + F}$ \\
\hline 2.7 & 0.28 & 9.1 & 31 & 46 & 68 & 45 & 199 & 120 & $\mathrm{~W} 1+0.5 \mathrm{~F}$ \\
\hline 3.0 & 0.38 & 11.0 & 41 & 60 & 75 & 40 & 154 & 113 & $\mathrm{~W} 2+0.5 \mathrm{~F}$ \\
\hline 0.352 & 0.051 & 1.508 & 5.22 & 6.501 & 6.544 & 2.550 & 16.515 & 6.627 & $\mathbf{L S D} \leq \mathbf{0 . 0 5}$ \\
\hline 0.683 & 0.091 & 3.212 & 11.67 & 15.22 & 10.79 & 5.021 & 40.521 & 15.820 & $\mathbf{L S D} \leq \mathbf{0 . 0 1}$ \\
\hline
\end{tabular}

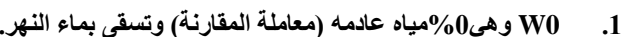
20 W2 W0+F 6 6.

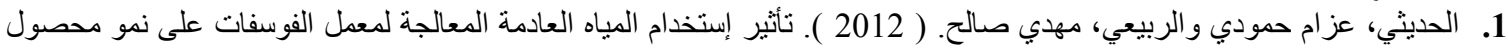

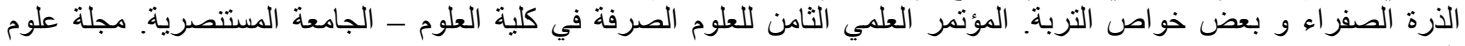

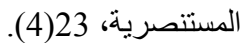
2. الحديثي، عزام حمودي و إلهام عبد الملك حسون. (a) 2011). استخدام المياه العادمة المعالجة في الري لعدة العدة مواسم زر اعية للمحافظة

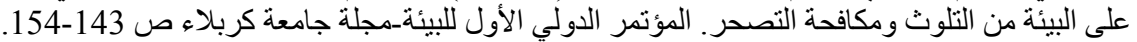

3. Abdullrzzak, mani. (2011). Membranes bioreactor sewage treatment for sustainable effluents reused MBR WWTPs

4. Bernd, F. (2009). Membrane BioReactors (MBR) For Dairy Effluent Treatment IDF World Dairy Summit. (Berlin).

5. Patsios, S.I. and A.J. Karabelas. (2007). Adavanced wastewater treatment processes: membrane bioreactors for water reuse "Modern Techno-logies for Water and Wastewater Treatment", Workshop, May 16, 2007

INTERREG III A / CARDS, Greece - F.Y.R.O.M.

6.Simon, j. (2010). The MBR Book: Principles and applications of membrane Bioreactors in Water and Wastewater Treatment

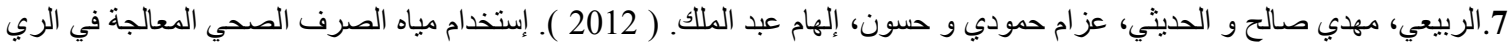

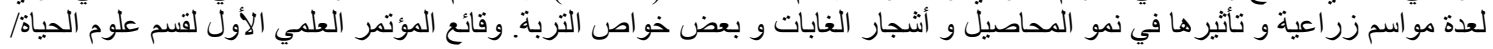

$$
\begin{aligned}
& \text { جامعة بغداد 387-397 }
\end{aligned}
$$

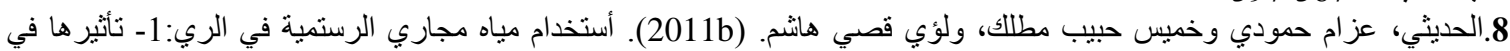

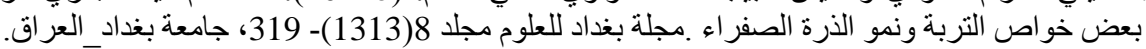

9. Angelakis, A.N.L, Bontoux, V. Lazarova. (2003).Challenges and prospective for water recycling and reuse in EU countries. Water Sci. Technol. Water Supply. 3 (4): 59-68. 
10. Abou- Seeda, M., El-Aila, H.I., Shehate, A. A. (1997). Waste water treatment for irrigation purposes 2. Sequential extraction of heavy metals in irrigated soils after one year. Mansoura- University- Journal of Agriculture Sciences ( Egypt ). 22(3) . P. 961- 973.

11. Hamdy, A. (1999). Sewage water for irrigation. Sustainable use of non-conventional water resources in the Mediterranean region. P.559-595.

12. Asano,T,F.L., Burton,H.L., Leverenz, R. Tsuchihashi, G. Tchobanoglous. (2006). Agricultural irrigation with reclaimed water on overview. Water Reuse. Issues, Technologies and Applications, Metcalf \& Eddy/AECOM. McGraw Hill, New York.

13.الحديثي، عزام حمودي خلف و عبد الرزاق، إبراهيم بكري. (2002). تأثير إضافة مياه المجاري على نمو النبات وتلوث التربة

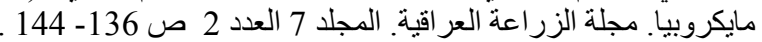

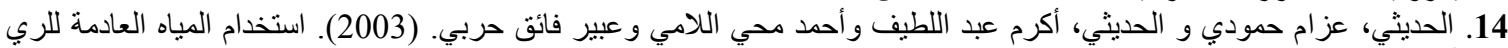

وتأثير ها في نمو الذرة الصفر اءو وبعض خو اص التربة.

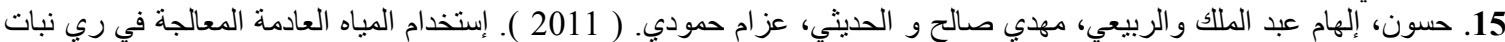

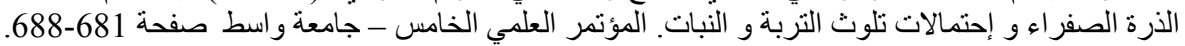

16.Capra, A.B. Scicolone. (2007). Recycling of poor quality urban wastewater by drip irrigationsystems. J.

Cleaner Prod. 15: 1529-1534.

17. أمل جركس. (2000). استخدامات مياه الصرف الصحي في الري. المجلة العربية لادارة مياه الري، صأ51 -58 المنظمة العربية

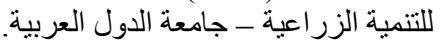

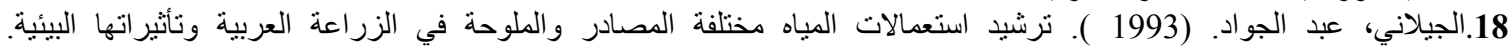

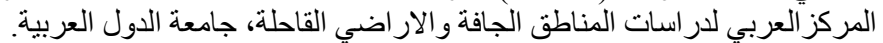

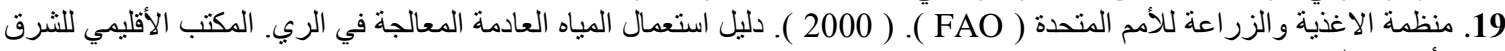
الأدنى - القاهرة - مصند.

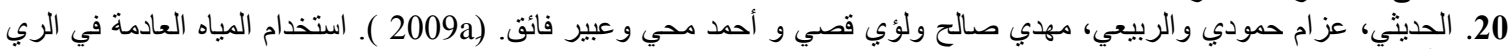

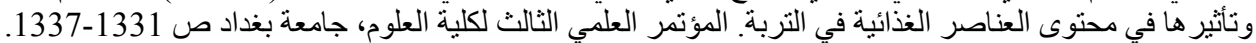

21.Ioukopoulos, B.I, K, Kalavrouziotis. (2008). Reuse of municipal reclaimed wastewater for the irrigation in oils and plants: Aitoloakarnania in Western Greece as an example. Fresenius Environ. Bull. 17 (4): 434-438.

22. الحديثي، عزام حمودي و عبد الرزاق، إبراهيم اهيمري. (2001 ). تأثير إضافة مياه مجاري الرستمية على محتوى العناصر الصغرى

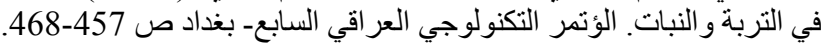

23.Keraita, B.N.P, Drechsel. (2004). Agricultural use of untreated urban wastewater in Ghana. C.A. Scott, N.I. Faruqui, L. Raschid-Sally (Eds.), Wastewater Use in Irrigated Agriculture, CABI Publishing, Wallingford, UK

$$
\begin{aligned}
& \text { 24.الحديثي، عز ام حمودي. (1987). تأثير اضافة مخلفات مجاري بغداد على نمو وانتاج الذرة الصفر اء واحتمالات تلوث التربة كيميائيا. }
\end{aligned}
$$

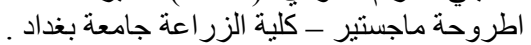

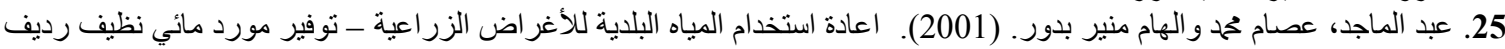

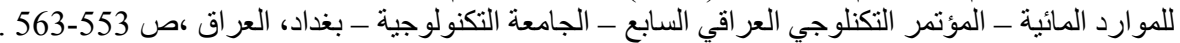

26.Lindsay, W.L., and W.A. Norvell. ( 1978 ). Devlopment of DTPA soil test for Zinc, iron, manganese and copper. Soil Sci. Sec. Amer. Proc. J. 42:421-428.

27.Page, A.L. (1982). Methods of Soil Analysis .Part2.Chemical and Microbiological properties. Amer . Soc. Agron. Madison. Wis. consin.

28.York, D.W.,R. Holden, B. Sheikh, L. Parsons. (2008). Safety and suitability of recycled water for irrigation of edible crops. Proceedings of the 23rd Annual WateReuse Symposium, WateReuse Association, Dallas.

29.Iglesias, R.E., Ortega. (2008). Present and future of wastewater reuse in Spain. Desalination. 218: 105119.

30.Richards. L.A. (1954). Diagnosis and improvementof saline and Alkali oil .14.15 USDA. HB No.60.

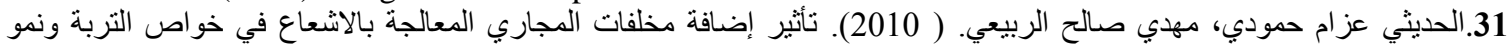

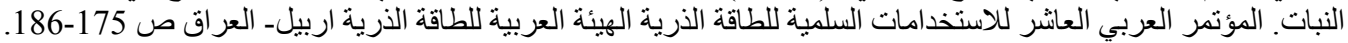

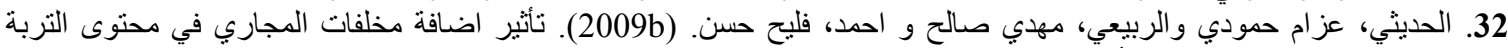

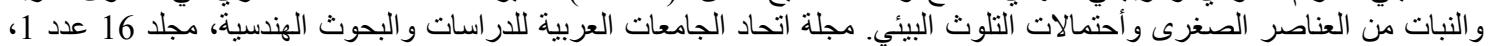

